\title{
Dual energy CT in gland tumors: a comprehensive narrative review and differential diagnosis
}

\author{
Francesco Gentili ${ }^{1} \wedge$, Susanna Guerrini ${ }^{1} \wedge$, Francesco Giuseppe Mazzei ${ }^{1}$, Ilaria Monteleone ${ }^{2}$, \\ Nunzia Di Meglio ${ }^{2}$, Letizia Sansotta ${ }^{2}$, Armando Perrella ${ }^{2}$, Sara Puglisi ${ }^{3}$, Massimo De Filippo ${ }^{3}$, \\ Paolo Gennaro $^{4}$, Luca Volterrani ${ }^{2} \wedge$, Maria Grazia Castagna ${ }^{5} \wedge$, Francesco $\operatorname{Dotta}^{6} \wedge$, \\ Maria Antonietta Mazzei ${ }^{2} \wedge$
}

${ }^{1}$ Unit of Diagnostic Imaging, Department of Radiological Sciences, Azienda Ospedaliero-Universitaria Senese, Siena, Italy; ${ }^{2}$ Unit of Diagnostic Imaging, Department of Medical, Surgical and Neuro Sciences and of Radiological Sciences, University of Siena, Azienda OspedalieroUniversitaria Senese, Siena, Italy; ${ }^{3}$ Unit of Radiology, Department of Medicine and Surgery, Azienda Ospedaliero-Universitaria di Parma, Parma, Italy; ${ }^{4}$ Department of Maxillofacial Surgery, University of Siena, Azienda Ospedaliera Universitaria Senese, Siena, Italy; ${ }^{5}$ Unit of Endocrinology, Department of Medical, Surgical and Neuro Sciences, University of Siena, Azienda Ospedaliero-Universitaria Senese, Siena, Italy; ${ }^{6}$ Unit of Diabetology, Department of Medical, Surgical and Neuro Sciences, University of Siena, Azienda Ospedaliero-Universitaria Senese, Siena, Italy Contributions: (I) Conception and design: F Gentili, MA Mazzei, F Dotta; (II) Administrative support: L Volterrani, MA Mazzei, M De Filippo, F Dotta, MG Castagna; (III) Provision of study materials or patients: F Gentili, S Guerrini, P Gennaro; (IV) Collection and assembly of data: I Monteleone, N Di Meglio, L Sansotta, S Puglisi; (V) Data analysis and interpretation: F Gentili, S Guerrini, A Perrella; (VI) Manuscript writing: All authors; (VII) Final approval of manuscript: All authors.

Correspondence to: Susanna Guerrini, MD. Unit of Diagnostic Imaging, Department of Radiological Sciences, Azienda Ospedaliero-Universitaria Senese, “Santa Maria alle Scotte” General Hospital, Viale Mario Bracci, 16, 53100 Siena, Tuscany, Italy. Email: guerrinisus@gmail.com.

\begin{abstract}
Dual energy CT (DECT)with image acquisition at two different photon X-ray levels allows the characterization of a specific tissue or material/elements, the extrapolation of virtual unenhanced and monoenergetic images, and the quantification of iodine uptake; such special capabilities make the DECT the perfect technique to support oncological imaging for tumor detection and characterization and treatment monitoring, while concurrently reducing the dose of radiation and iodine and improving the metal artifact reduction. Even though its potential in the field of oncology has not been fully explored yet, DECT is already widely used today thanks to the availability of different CT technologies, such as dual-source, singlesource rapid-switching, single-source sequential, single-source twin-beam and dual-layer technologies. Moreover DECT technology represents the future of the imaging innovation and it is subject to ongoing development that increase according its clinical potentiality, in particular in the field of oncology. This review points out recent state-of-the-art in DECT applications in gland tumors, with special focus on its potential uses in the field of oncological imaging of endocrine and exocrine glands.
\end{abstract}

Keywords! Pancreas; ovary; dual energy CT; salivary glands; mammary gland

Submitted Jun 01, 2020. Accepted for publication Sep 07, 2020.

doi: $10.21037 / g s-20-543$

View this article at: http://dx.doi.org/10.21037/gs-20-543

^ ORCID: Francesco Gentili, 0000-0003-2492-5844; Susanna Guerrini, 0000-0001-7875-3342; Luca Volterrani, 0000-0001-9609-1116X; Maria Grazia Castagna, 0000-0002-2457-1751; Francesco Dotta, 0000-0003-4947-7478; Maria Antonietta Mazzei, 0000-0001-6778-6894. 


\section{Introduction}

The terms dual-energy CT (DECT) refers to the acquisition of CT attenuation data deriving from two photon energies, in this way the attenuation differences between various materials could provide their characterization (1-4). Despite the fact that the role of DECT in diagnostic imaging was described in the late 1970, only in the last years, thanks to the emerging technologies, it has become possible to perform two acquisitions almost simultaneously at different tube voltage, avoiding misregistration artefacts between the two imaging sets $(5,6)$. Independently of the technical approach to obtain DECT data (dualsource, single-source rapid-switching, single-source sequential, single-source twin-beam and dual-layer), a set of monochromatic images can be extracted and tissues characterized through a decomposition algorithm (7). Furthermore, the increased attenuation of iodine at a low energy level, close to the $\mathrm{k}$ edge of this material $(33.2 \mathrm{keV})$, improves the identification of vascularised lesions and the contrast between a hypervascular or hypovascular lesion and the normally enhancing surrounding parenchyma $(7,8-13)$. Material decomposition algorithm allows the generation of additional set of images such as iodine images and virtual non contrast (VNC) images (14). Iodine specific maps allow to quantify iodine uptake in each voxel in order to detect even a little enhancement in a lesion, parenchyma or in the wall of hollow organs (15-19). Thanks to the new algorithms VNC images have a contrast to noise ratio (CNR) and Hounsfield Unit (HU) accuracy similar to true non-contrast (TNC) ones; in this way, a true scan before administration of contrast medium can be avoided, reducing the radiation dose to the patient $(7,20,21)$. These tools have been investigated in different fields, improving diagnostic possibilities $(22,23)$. In addiction, the sensitivity of DECT for iodine permits the reduction of amount and concentration of intravenous contrast media, since monoenergetic images at lower $\mathrm{keV}$ (ranging from 50 to 70 ), substantially increase iodine attenuation and might improve image quality (IQ) if contrast dose is reduced, finally reducing kidney injuries especially in oncologic patients $(6,7,13)$. Last but not least DECT, the use of the high-energy (high $\mathrm{keV}$ ) monoenergetic data set can help in streak artifact reduction, that could be a common eventuality in the CT evaluation of salivary glands tumour due to metallic dental implants or dental amalgam or in case of dorsal or lumbar spine stabilization $(24,25)$. The aim of this work is to provide a comprehensive review of the application of DECT about tumors of exocrine and endocrine glands. We present the following article in accordance with the NARRATIVE REVIEW reporting checklist (available at http://dx.doi.org/10.21037/gs-20-543).

\section{Endocrine glands}

\section{Adrenal glands}

The most common adrenal lesion observed on CT, often incidentally, is the adenoma $(26,27)$. The frequency of these lesions in the general population is close to $5 \%$ and it is fundamental to distinguish them from metastatic and other malignant lesions $(26,28)$.

In this regard, Helck et al. (29), in 2013, identified retrospectively 51 patients with incidental adrenal lesions in a cohort of 457 DECT exams of the abdomen, performed only in the portal venous phase. Forty-eight adrenal lesions turned out to be benign and 9 malignant.

By using the threshold of $10 \mathrm{HU}$ on VNC images, the sensitivity, specificity and accuracy for differentiating of between benign adenomas and malignant lesions were $73 \%$, $100 \%$ and $81 \%$ respectively. However many lipid-poor adenomas showed a density major than $10 \mathrm{HU}$, making impossible to distinguish them from malignant lesions.

In 2014, Botsikas et al. (30) analysed 20 patients with one or more adrenal lesions, performing DECT scans without contrast and post contrast, both in the portal and late phases (15 min). The authors found a significant difference between HU of adrenal lesions on TNC images and VNC images calculated from portal venous phase $(8.58 \pm 15.2 \mathrm{vs}$. $12.6 \pm 14.9 \mathrm{HU}$ ) whereas TNC and VNC images derived from late phase showed no significant difference each other. The authors concluded that DECT portal phase can be sufficient for diagnosing an adenoma only in the case of $\mathrm{HU}$ value lower than 10 , otherwise a late scan at 15 minutes should be performed, evaluating the wash-out pattern, for solving the diagnostic dilemma.

Before, in 2010, Gupta et al. (31) investigated 31 patients with adrenal lesions who underwent DECT scans without contrast. Twenty of the 31 nodules were classified as lipidrich adenomas, 6 as lipid-poor adenomas and 5 as metastatic lesions. The authors analysed the attenuation changes of the lesions between 140 and $80 \mathrm{keV}$ and found a significant difference between all adenomas (mean reduction of $0.4 \pm 7.1 \mathrm{HU}$ ) and metastatic lesions (mean increase of $9.2 \pm 4.3 \mathrm{HU}$ ). The authors concluded that a decrease in attenuation from 140 to $80 \mathrm{keV}$ is indicative of adenoma with $100 \%$ of specificity and positive predictive value but 

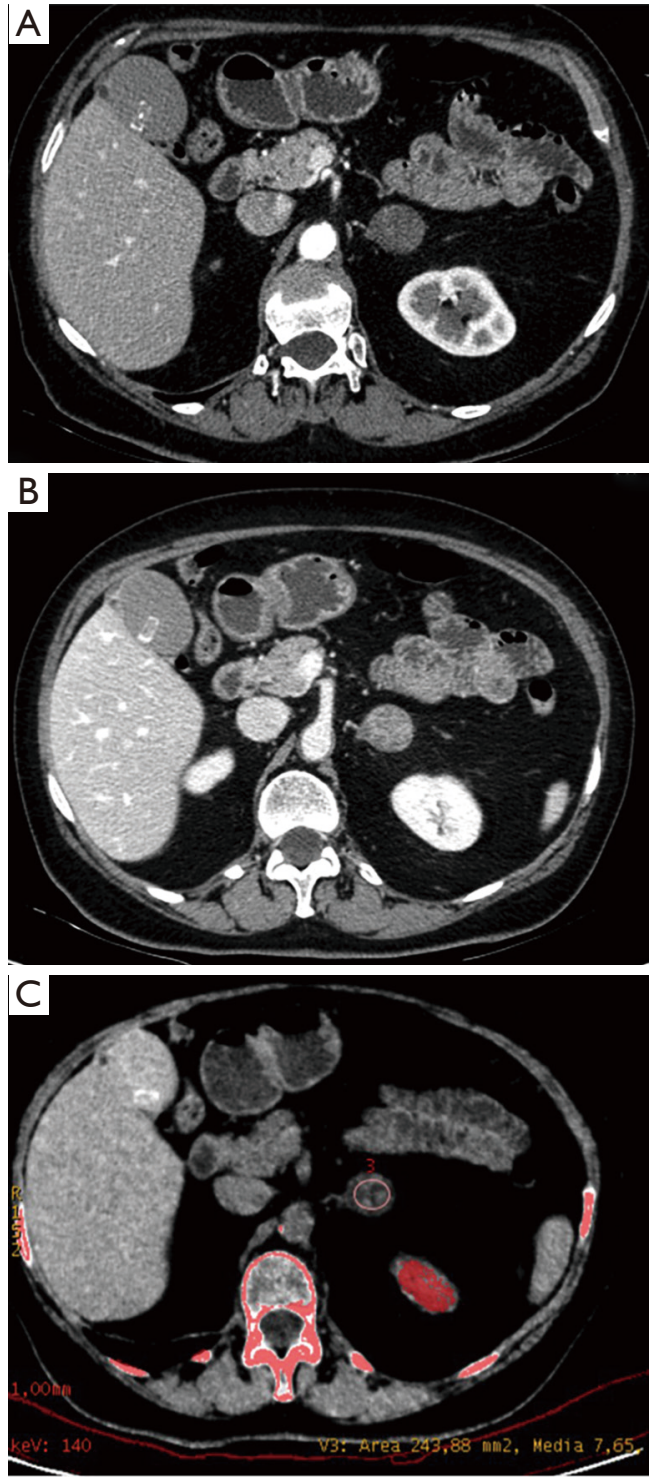

Figure 1 Lipid-poor adenoma: incidental adrenal lesion observed on post-contrast CT images (A,B), was correctly demonstrated to be an adenoma with VNC images, derived from late phase, showing HU values lower than $10(\mathrm{C})$.

with low sensitivity and negative predictive value $(50 \%$ and $28 \%$ respectively) (Figure 1).

\section{Pancreas}

Pancreatic ductal adenocarcinoma (PDAC) is the fourth cause of cancer-related deaths in United States, with a very low 5 -year overall survival (32). Surgical resection is the only therapeutic option, hence an accurate detection, characterization and staging with CT or MR imaging is essential (33). PDAC is usually hypovascular, if compared to normal parenchyma, due to the high percentage of associated desmoplasia and fibrosis (34). However, on CT, a significant proportion of focal lesions are slightly hypo- or isodense to the pancreatic parenchyma, making difficult their detection (35). For this reason, many studies have investigated the feasibility of virtual monoenergetic series and iodine maps, in terms of signal and CNR, in pancreatic cancer evaluation (35-41). Bhosale et al. (38), in 2015, compared CNR and signal to noise ratio (SNR) between DECT and $120 \mathrm{kVp}$ CT images in pancreatic phase, in patients with PDAC. Forty patients were studied with DECT and 38 with single energy CT. Monochromatic images were reconstructed at 50, 60, 70 and $80 \mathrm{keV}$ values; Iodine maps provided a significant higher CNR and SNR for PDAC than any other image set. Moreover, iodine images were rated as the best for detecting primary cancer. The $70 \mathrm{keV}$ series and $120 \mathrm{kVp}$ single energy images were qualitatively judged as the best to identify tumor extension and vascular invasion, due to lower noise.

Beer et al. (37), in 2019, analyzed 45 patients with PDAC who underwent abdominal DECT staging with both pancreatic parenchymal phase and portal venous phase. Different quantitative and qualitative parameters were extracted on virtual monochromatic series. Tumor linedensity analysis revealed the highest contrast difference between tumor and normal pancreatic tissue at $40 \mathrm{keV}$ monoenergetic reconstruction. In the same way, $40 \mathrm{keV}$ monoenergetic images provided the highest CNR and SNR for PDAC in the pancreatic parenchymal phase. Iodine uptake of PDAC was significantly lower than normal pancreatic tissue, both in pancreatic phase and portal venous phase $(2.0 \pm 1.1$ vs. $4.8 \pm 1.3 \mathrm{mg} / \mathrm{dL}$ and $2.1 \pm 1.1$ vs. $4.4 \pm 1.0 \mathrm{mg} / \mathrm{dL}$, respectively). Subjective image quality was assessed using a 5 -point Likert scale and $40-50 \mathrm{keV}$ images were considered the best for evaluating PDAC. El Kayal et al. (35), in 2019, analyzed 61 patients with pancreatic lesions of different origins, who underwent DECT scans in portal venous phase. The authors found that lesion conspicuity was significantly higher on monoenergetic images at $40 \mathrm{keV}$, iodine density and iodine overlay reconstructions. Subjective analysis revealed a significantly higher lesion conspicuity and reader confidence using monoenergetic images at low $\mathrm{keV}$ levels, if compared to polychromatic images. Low $\mathrm{keV}$ series even detect small lesions with only little contrast to the surrounding pancreatic tissue. However quantitative 
data still did not allow a confident discrimination between PDAC and other hypodense lesions. Chu et al. found that iodine images were helpful in determining the solid or cystic nature of a lesion, improving also the evaluation of relationships between tumor, main pancreatic duct and vessels (42). Considering pancreatic lesions different from PDAC, virtual monoenergetic images and iodine maps may better indicate the complexity of cystic lesions $(39,43,44)$.

DECT has been also investigated for the differentiation of serous and mucinous cysts. A iodine density $>0.325 \mathrm{mg} \cdot \mathrm{mL}^{-1}$ in the arterial phase was found to be predictive of mucinous cystic neoplasm with an AUC of 0.68 (36).

\section{Ovary}

Only a few works have investigated DECT for diagnosing ovarian cancer. This neoplasia is the most lethal among pelvic cancers and more than $60 \%$ of patients already present metastatic peritoneal spread at the time of diagnosis $(45,46)$. Imaging plays a fundamental role both in the ovarian lesion characterization and in the definition of peritoneal spread $(47,48)$ Pang et al. (49) analyzed 22 patients with suspected ovarian tumors. An unenhanced scan was followed by post-contrast arterial and venous phases. Monochromatic images were reconstructed. Then $\mathrm{HU}$ at $40 \mathrm{keV}$, iodine concentration (IC) and water concentration were measured into the most homogeneous solid area of the lesion. HU curve slope between 40 and 100 $\mathrm{keV}$ was calculated. At final pathology 11 lesions proved to be malignant and 11 benign. Among the malignant lesions, 3 were serous adenocarcinomas, 3 mucinous adenocarcinomas, 3 mixed cystadenocarcinomas and 2 metastatic tumors. The benign group included 2 fibromas, 3 serous cystadenomas, 5 mucinous cystadenomas and 1 sclerosing stromal tumor. A statistical significant difference in $\mathrm{HU}$ at $40 \mathrm{keV}$ and IC was found between malignant and benign lesions; in particular values were higher in the first group. In the arterial phase the best parameter was IC (AUC of 0.90 , sensitivity $88.9 \%$, specificity $94.7 \%$, threshold value $1.09 \mathrm{mg} / \mathrm{dL}$ ). No significant difference was found in water concentration. The higher IC within malignant tumors reflected neovascularization phenomena with tortuous vessels and arteriovenous fistulas $(50,51)$.

DECT seems to be helpful for differentiating simple cysts from cystic tumors, given the increased conspicuity of cyst wall and septa at low $\mathrm{keV}$ images. In metastatic patients, low $\mathrm{keV}$ images improve the visualization of peritoneal carcinomatosis and in particular of perisplenic, perihepatic and subdiaphragmatic peritoneal implants (52) (Figure 2).

\section{Thymus}

DECT has been investigated for the diagnosis of suspected neoplastic lesions in thymic lodge. Yan et al. (53), in 2018, analyzed 57 patients with low-risk thymoma $(\mathrm{n}=16)$, highrisk thymoma $(n=15)$, thymic carcinoma $(n=14)$, and thymic lymphoma $(n=12)$. All the DECT exams of the thorax were performed in arterial and venous phases. In postproduction virtual monoenergetic series and iodine maps were generated. Iodine-related $\mathrm{HU}$ value in the venous phase showed the best accuracy in differentiating low-risk thymomas from high- risk thymomas or thymic carcinoma (AUC of $0.893,75.0 \%$ sensitivity, $89.7 \%$ specificity at the cutoff value of $34.3 \mathrm{HU}$ ). In the venous phase IC showed the best accuracy in differentiating low-risk thymomas from thymic lymphoma (AUC of 0.969 , 87.5\% sensitivity, 100\% specificity at the cutoff value of $1.25 \mathrm{mg} / \mathrm{mL}$ ).

Few years before, in 2016, Chang et al. (54) investigated the possibility of DECT in differentiating low-risk thymoma from high-risk thymoma and thymic carcinomas. At final histopathology 23 tumors proved to be low-risk thymomas, 5 high-risk thymomas and 9 thymic carcinomas. Iodine-related HU and IC were significantly different between low-risk thymomas, high-risk thymomas and thymic carcinomas (median: 29.78 vs. 14.55 vs. $19.95 \mathrm{HU}$, $\mathrm{P}=0.001$ and 1.92 vs. 0.99 vs. $1.18 \mathrm{mg} / \mathrm{mL}, \mathrm{P}<0.001$, respectively).

Even though the significant results of DECT quantitative parameters, it is fundamental to take into account qualitative features for differential diagnosis: lymphadenopathies are not usually associated with low and high risk thymoma whereas they are visible in a low percentage (about $20 \%)$ of thymic carcinomas $(53,55)$. Moreover low-risk thymomas have smooth or lobulated boundaries whereas thymic carcinomas have irregular contours in a significant percentage of cases (54). Metastases can be present in case of high-risk thymoma and thymic carcinoma and the pleura is the most common site $(55,56)$. In these patients, pleura and diaphragmatic pillars should be attentively analyzed in order to avoid to underreport metastatic pleural lesions or mistake them for pleural plaques (56-60). DECT may be integrated with texture analysis and CT perfusion, which are promising for grading and staging neoplasms (61-68). MRI should be performed for characterizing a thymic lesion only in selected cases, such as the distinction between 

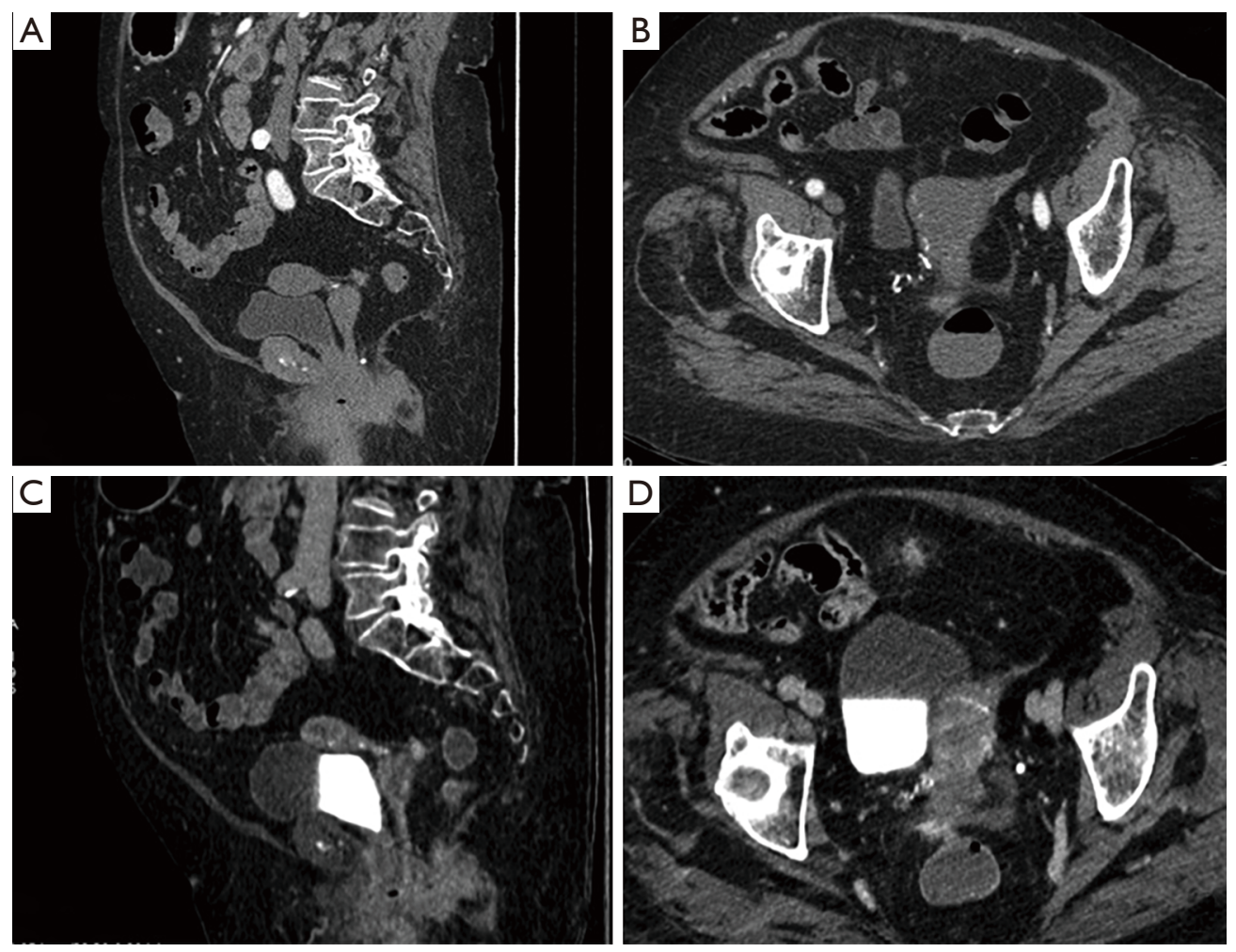

Figure 2 In a 63-year-old patient with ovarian cancer and peritoneal carcinomatosis (A,B), DECT images (C,D) are helpful for detecting a peritoneal implant in rectouterine pouch, thanks to the increased conspicuity at low keV images. In metastatic patients, low keV images improve the visualization of peritoneal carcinomatosis especially in cases without free peritoneal fluid.

a small thymic neoplasm and an atypical thymic hyperplasia, exploiting dual gradient-echo in-phase and opposed-phase sequences. MR lymphangiography may be used in the suspicion of a lesion of lymphatic origin (69-73).

\section{Thyroid}

Thyroid cancer is the most commonly diagnosed cancer in young women; it has shown a significant increase in the United States, Canada, Sweden, and Britain in the last decade, mainly due to environmental factors (74). Although thyroid cancer is one of the most curable malignancies at early stages and only a few nodules are malignant (about 5-7\%), differential diagnosis can be a challenge for ultrasonography and fine needle aspiration biopsy, the latter considered as the tool of choice for the diagnosis (75-79). DECT has been investigated in some works for characterizing thyroid nodules; this technique can evaluate at the same time the extension to adjacent structures and lymph nodes in case of malignancy (80).
In 2015, Li et al. (80) analyzed 122 thyroid nodules. All the patients underwent CT scans of the neck in GSI mode with the fast tube voltage switching between $80 \mathrm{kVp}$ and $140 \mathrm{kVp}$. A CT scan without contrast was followed by post contrast scans. An automatic trigger software, SmartPrep, was employed to determine the time for the arterial and venous phase. For each nodule, both endogenous IC in the scan without contrast and exogenous IC after contrast medium injection were recorded. Moreover, in postcontrast phases, exogenous IC was normalized to IC of common carotid artery (iodine ratio). Eighty-seven nodules proved to be benign and 35 malignant: 27 papillary carcinomas; 1 medullary carcinoma; 4 poorly differentiated carcinomas; 2 anaplastic carcinomas; 1 metastatic squamous cell carcinoma.

In the scans without contrast endogenous IC was significantly lower in the malignant group than in the benign (AUC 0.7 , optimal threshold $0.68 \mathrm{mg} / \mathrm{mL}$, sensitivity $74.3 \%$ and specificity $66.7 \%$ ). No significant differences were found between benign and malignant 
nodules in arterial phase. In venous phase, iodine ratio of malignant group was significantly lower than benign (AUC 0.88 , threshold 0.38 , sensitivity $76.9 \%$, specificity $80 \%$ ). Multivariate analysis considering solitary morphological pattern, irregular margin, presence of calcification and edge interruption determined an AUC of 0.87 , sensitivity $77.1 \%$ and specificity $84.5 \%$ for non-contrast phase and 0.94 , sensitivity $84.6 \%$, specificity $88 \%$ for venous phase, in distinguishing malignant from benign lesions. The lower iodine concentration in thyroid cancer can be explain by the disappearance of almost normal follicular cells, which are replaced by cancer cells, unable to uptake iodine.

In 2016, Gao et al. (81) analyzed 26 malignant and 33 benign nodules. The authors performed a pre-contrast scan and one post-contrast scan by using the same machine of Li et al. (80). Similarly, they found a significant difference between malignant and benign nodules in the scans without contrast (AUC 0.95 , threshold $0.67 \mathrm{mg} / \mathrm{ml}$, sensitivity $92.3 \%$, specificity $88.5 \%$ ). On the contrary they found a significant higher iodine concentration in malignant nodules than in benign, in the post contrast scans; however the timing of post-contrast phase was not specified. Recently, in 2018, Lee et al. (82) enrolled 76 thyroid nodules (46 papillary carcinomas, 17 benign and 13 cysts). CT scans were performed only in the arterial phase (fixed delay of $40 \mathrm{sec}$ ) by using a single source, dual-layer detector DECT. The authors found a significant lower iodine ratio (between the nodule and the normal parenchyma) in malignant than in benign nodules. DECT has been also investigated to evaluate metastasis to cervical lymph nodes in papillary thyroid cancer with encouraging results. Liu et al. (83), in 2015 , analyzed 45 patients ( 21 cases of papillary cancer and 24 benign diseases) with a total of 175 lymph nodes (63 metastatic, 54 excised benign and 58 unexcised benign). The exams were acquired by using a rapid $\mathrm{kVp}$ switching mode, in three phases: unenhanced, arterial and venous phase. The slope of the HU attenuation curve of each lymph node was calculated as the difference between the CT value at $40 \mathrm{keV}$ and that at $70 \mathrm{keV}$ divided by the energy difference. IC of each lymph node was normalized to that of left common carotid artery.

No significant difference in DECT parameters was found between benign and metastatic lymph nodes on unenhanced images. The mean arterial and venous HU slope and IC were significantly different between benign and metastatic lymph nodes, with the highest accuracy for HU slope in the venous phase (a threshold of $>5.10$ identifies malignant lymph nodes with a sensitivity of $62 \%$ and specificity of
91.1\%, AUC of 0.791). Moreover the combination of HU slope in the venous phase and normalized IC in arterial phase reaches an AUC of 0.811 .

The higher HU slope and IC of metastatic lymph nodes can be explained by the increase in the number of blood vessels and change in vascular pattern tumor-associated $(84,85)$.

\section{Parathyroid}

DECT has been investigated for localizing a parathyroid adenoma in patients with the suspicion of primary hyperparathyroidism. Seyednejad et al. (85), in 2016, compared the two imaging modalities commonly used for preoperative identification of parathyroid tumor (PT), ultrasound and technetium-99m sestamibi imaging with single photon emission CT, with DECT. DECT exams were performed, before and after administration of contrast medium. The non-contrast phase was considered useful in trying to distinguish between a PT and thyroid gland, since the tumor does not contain iodine, in contrast to thyroid gland; images were reconstructed at $50 \mathrm{keV}$, closer to the K-edge of iodine, in order to optimize attenuation difference between thyroid tissue and PT. The post contrast phase was chosen in order to emphasize the attenuation difference between PT, thyroid gland and adjacent lymph nodes $(86,87)$.

On post contrast DECT images, PT was defined by the presence of a lesion enhancing greater than the muscle, with an attenuation lower than thyroid gland on noncontrast images, both in a typical or ectopic location. The final population was composed of 21 patients with 18 cases of solitary adenoma, 4 cases of double adenoma and a single case of parathyroid hyperplasia. The accuracy of each preoperative imaging modality, based on postoperative histopathology was: $58 \%$ for US, $75 \%$ for CT-MIBI and $75 \%$ for DECT (86).

In 2015, Roskies et al. (88) investigated 29 patients with primary hyperparathyroidism and non-localized parathyroid adenoma. The scans were performed after the injection of contrast medium with a delay of 25,55 and $85 \mathrm{sec}$. Images were reconstructed into virtual monoenergetic series, ranging from 140 to $40 \mathrm{keV}$. Potential adenomas were identified on the basis of qualitative features, such as location, presence of a fat plane between the adenoma and thyroid, size, shape and polar arteries. Quantitative DECT features were analyzed. Virtual unenhanced images were not found helpful since the intrinsic iodine content of the thyroid gland is also 
suppressed, making difficult to distinguish the adenoma from thyroid gland. A significant difference was found between lymph nodes and adenomas attenuation at low energies on the arterial phase (delay of $25 \mathrm{sec}$ ) whereas no significant difference was found in the other phases. In particular, most adenomas showed a strong enhancement in arterial phase with a fast washout pattern (88).

\section{Exocrine glands}

\section{Mammary gland}

DECT has been investigated for evaluating breast cancer in some recent works, given that invasive neoplastic lesions are usually hypervascular compared to the normal parenchyma, with a significant iodine uptake (89-92). The advantage of DECT, if it proved reliable for local staging, would be the possibility to assess primary breast lesions and search for distant metastases, by performing a single diagnostic examination. Among the disadvantages it should be remembered the radiation dose, that can be high in case of multiple post-contrast phases, and the low accuracy for evaluating carcinomas in situ, if compared to MR $(93,94)$.

Metin et al. (92), in 2019, investigated the role of this technique for improving conspicuity of primary breast cancers. The authors analyzed 29 patients with 39 histopathologically proven breast tumors. The exams were performed in a single post-contrast phase (50 sec after contrast agent injection). Readers visually graded the conspicuity of lesions on virtual monochromatic images. Subjective score proved the highest conspicuity of breast lesions at $40 \mathrm{keV}$ and quantitative parameters confirmed this result. Moon et al. (89), in 2019, tried to correlate tumor conspicuity on low virtual monoenergetic images with prognostic biomarkers in 64 patients with breast cancers. The exams were performed on a dual-layer spectral detector CT in arterial and delayed phase (90 sec after contrast medium injection). The authors found that conspicuity score and attenuation values of cancers in delayed phase were significantly higher to that on arterial phase with an AUC for distinguishing cancers from other lesions of 0.817 . However, conspicuity score and attenuation values were significantly higher on arterial phase in tumors with high histologic grade, ER negativity, PR negativity, HER 2 positivity and Ki67 positivity. On the contrary, no significant difference was found on delayed phase between low and high risk tumors, probably because the arterial phase best reflects difference in neovascularization phenomena. Similarly, Okada et al. (91) evaluated breast cancer conspicuity on virtual monoenergetic images; however the series were reconstructed also by using a new algorithm (Monoenergetic Plus), that seems to reduce the increased noise in low $\mathrm{keV}$ images. Forty-two patients underwent contrast-enhanced CT by using a dual-source scanner with pre and post contrast scans (40, 90, 120 and $150 \mathrm{sec}$ after contrast medium injection) for a total of 5 phases. In all images series, CNR was highest at $40 \mathrm{keV}$, by using Monoenergetic Plus. However, the images at $50 \mathrm{keV}$ reconstructed with Monoenergetic Plus were defined the most clinically useful considering the overall image quality. It should be noted the unjustifiable high radiation dose derived from the multiple scans and that the authors did not specify the peak enhancement phase used for quantitative measures.

Inoue et al. (90), in 2020, enrolled 28 patients with breast cancer. The exams were performed with a dual-layer CT in a single post-contrast phase (70 sec from contrast medium injection). The authors compared CT attenuation values, image noise and CNR between virtual monoenergetic images and conventional CT images. They found that breast cancer attenuation increased as the energy level decreased and similarly image noise increased as the energy level decreased. CNR of $40 \mathrm{keV}$ images was significantly higher than those of conventional images, demonstrating the superiority of DECT in evaluating primary breast cancer.

Volterrani et al. (93) analysed 64 breast cancer lesions in 31 patients, investigating the possibility of DECT for local staging and identifying tumor lesions, trying to distinguish different histotypes. All the exams were performed with a 64-slice scanner in post contrast late arterial phase (45-50 sec from contrast medium injection). Virtual monochromatic images were reconstructed at 40 and $70 \mathrm{keV}$ and iodine maps were generated. DECT identified 67 hypervascular lesions (all the invasive cancers, 8 of 10 carcinomas in situ and 5 nonmalignant lesions) versus 64 tumor lesions on histology. T category was correctly identified in $85 \%$ of cases. Iodine maps proved to be the best series for differentiating tumor histotypes. In particular lesion IC showed an AUC of 0.968 (sensitivity $94.9 \%$, specificity $93.0 \%$, threshold $>1.70 \mathrm{mg} / \mathrm{mL}$ ) for distinguishing invasive cancers from other lesions. The ratio between iodine concentration of breast lesions and normal breast parenchyma showed that a threshold $>6.13$ was the best for distinguishing invasive ductal carcinoma from other lesions (sensitivity, 87.0\%; specificity, $81.1 \%$; 


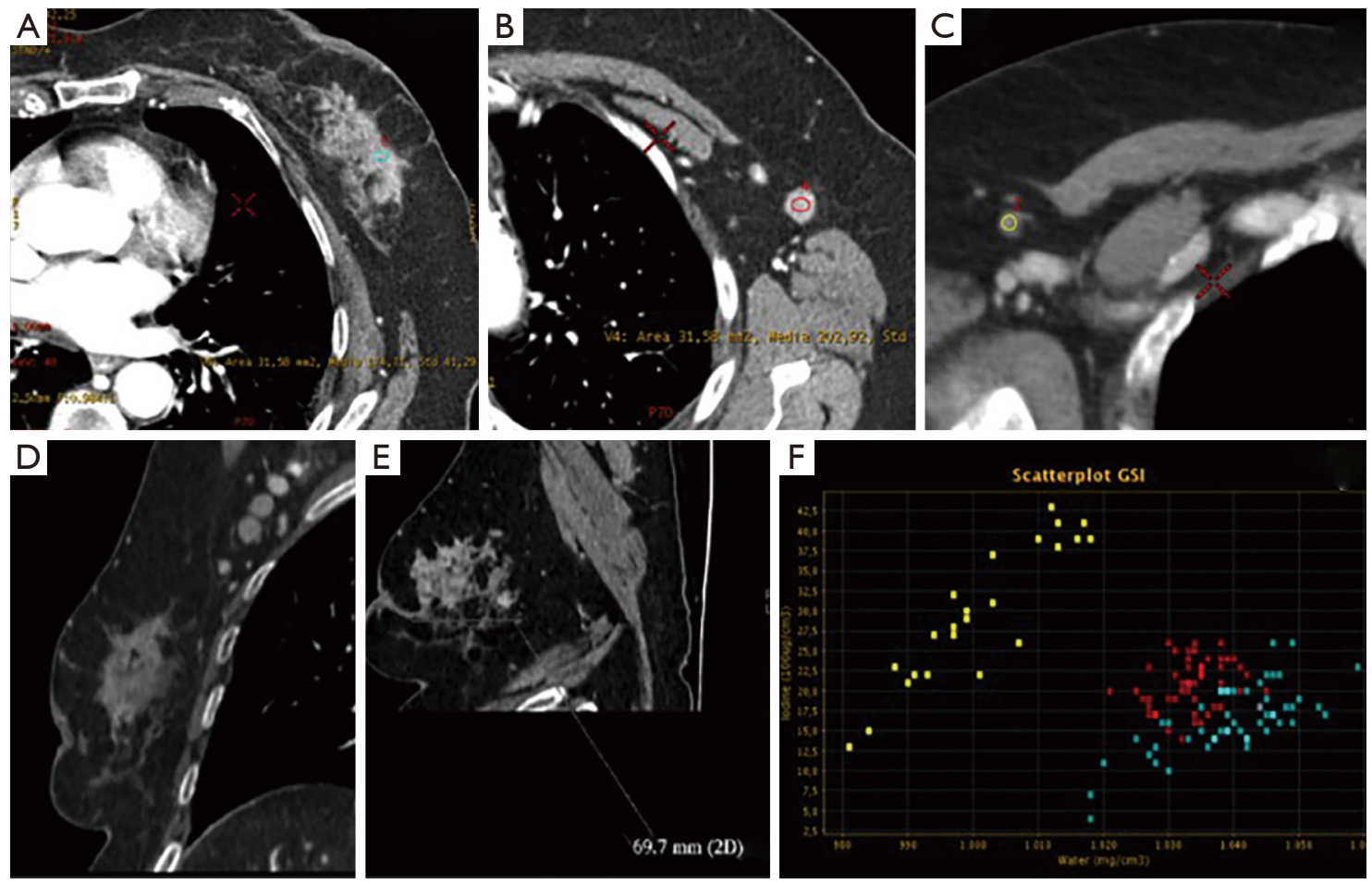

Figure 3 Seventy-eight-year-old patient with lobular carcinoma of left breast with a metastatic left axillary limphonodes (A,B,C,D,E). As you can see from the scatterplot $(\mathrm{F})$, the concentration of iodine in breast lesions outlined with blue ROI and in the metastatic lymphonode marked with red ROI, are overlapping while a heathy lymphonode in the right axilla, marked with yellow ROI shows a different distribution of iodine.

AUC value, 0.914).

DECT has been exploited in the attempt to identify the status of axillary sentinel lymph nodes (SLNs). In fact, imaging characterization of SLNs would be desirable for treatment planning and decision making, reducing the risk of post-surgical lymphedema derived from node biopsy $(95,96)$.

In this regard, Zhang et al. (97) analysed 193 patients, who underwent dual-phase post-contrast DECT, by using a 64 multidetector row CT. The exams were performed in arterial and venous phases. Two readers analysed morphologic and quantitative DECT parameters of lymph nodes, such as IC and HU slope of spectral curve between 70 and $40 \mathrm{keV}$. Parameters were also normalized to that of the aorta in each phase. At histopathological analysis 55 SLNs proved to be metastatic and 138 benign. Among the morphologic criteria, the highest accuracy $(81 \%)$ was achieved by the cortex status of lymph node (abnormal $v s$. normal), with a high specificity $(93.6 \%)$ but with a very low sensitivity (31\%) and an AUC of 0.62 .

Among the quantitative parameters, arterial and venous phase HU slope and normalized IC of metastatic SLNs were significantly higher than those of benign SLNs. The HU slope in venous phase showed the highest diagnostic accuracy $(90.5 \%$, sensitivity $66 \%$, specificity $97.7 \%$, AUC of 0.88 ). Therefore dual-energy CT seems promising for preoperative identification of pathological SLNs in breast cancer patients (Figure 3).

\section{Salivary glands}

Differential diagnosis between benign and malignant tumors of salivary glands is a diagnostic challenge that strongly influences the surgical approach $(98,99)$. In numerous studies, multiphase contrast-enhanced CT has been described to be reliable for diagnosing parotid tumors, even if the radiation dose is very high (100-102).

In the CT evaluation of salivary glands tumour DECT has numerous advantages: (I) differential diagnosis between benign and malignant lesions (complete lack of iodine in a parotid suggests the diagnosis of a cyst and further confirmatory ultrasound examination may be avoided); 

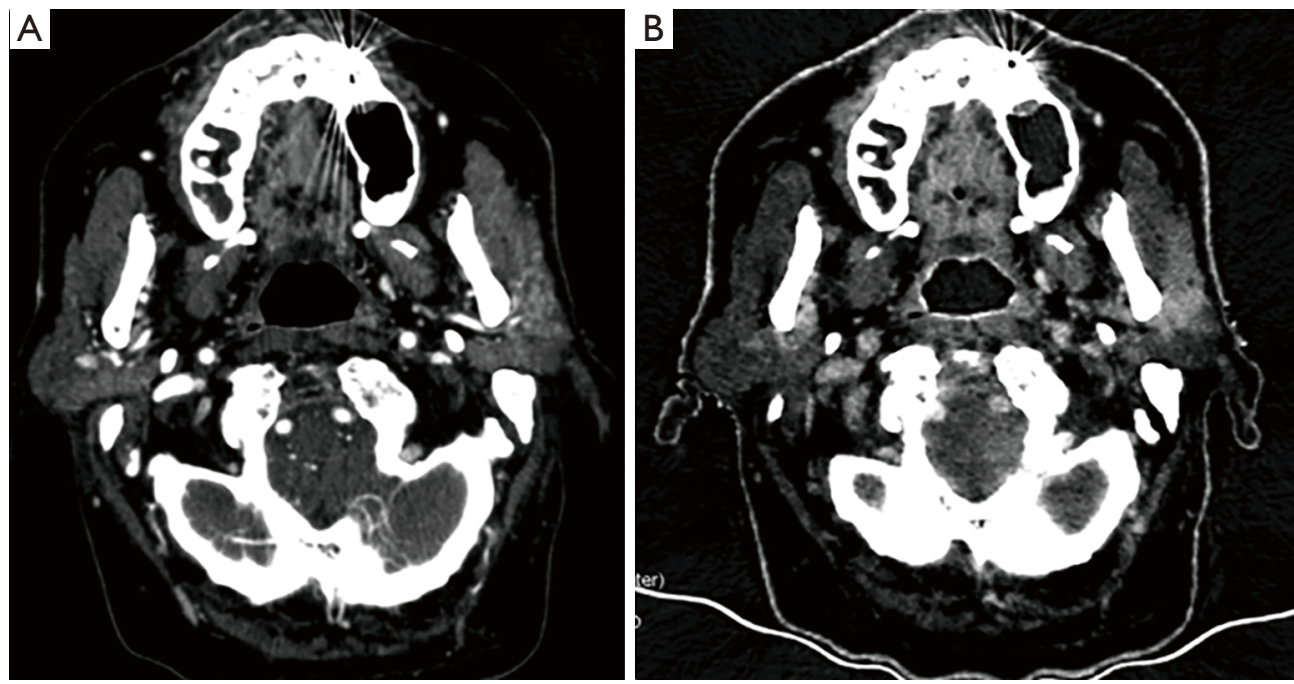

Figure 4 Sixty-five-year-old patient with a left parotid gland tumour. At conventional CT image (A), the tumours' margins are not clearly delineated; DECT image (B) allows a better visualisation of the tumour, inseparable from the left masseter muscle.

(II) improvement of detection of benign salivary gland tumours, by utilization of the low-energy monoenergetic images (pleomorphic adenomas may not be appreciated on contrast-enhanced conventional CT owing to poor enhancement and overall low density of the parotid gland); (III) reduction of streak artefacts that could often cause an image degradation in CT of the head and neck due to metallic dental implants and dental amalgam used for filling up the dental caries that is composed of metal. These streak artefacts often extend to the salivary glands could cause suboptimal evaluation, however since they result from photon starvation (less number of photons reaching the detector), utilization of the high-energy data set could help in streak artefact reduction. The same data set of images could allow a better evaluation of enhancing tumour with intracranial spread via the skull base foramina thanks to the reduction of artefacts deriving from bony skull.

Li et al. (103), in 2018, investigated 50 patients with suspected parotid tumours, who underwent postcontrast DECT with a scan delay of $45 \mathrm{sec}$ from contrast medium injection. Finally, 53 tumours were confirmed at histopathological analysis: 12 pleomorphic adenomas (PAs), 24 Warthin tumours (WTs) and 16 malignant tumours (MTs). Monochromatic images, water and iodine maps were reviewed and quantitative DECT parameters (iodine and water concentration, slope of $\mathrm{HU}$ between 100 and $40 \mathrm{keV}$ ) were measured. IC was also normalized to common carotid artery. Significant differences were found between the 3 groups of tumours; in particular all the parameters were highest in WTs, followed by MTs and PAs. The optimal thresholds of IC, normalized IC and HU slope were $1.46 \mathrm{mg} / \mathrm{mL}, 0.20$ and 1.72 for distinguishing WTs from MTs with a sensitivity of $91.7 \%, 95.8 \%$ and $91.7 \%$, and a specificity of $89.3 \%, 85.7 \%$ and $89.3 \%$, respectively. The corresponding threshold for distinguishing MTs from PAs were $0.91 \mathrm{mg} / \mathrm{mL}, 0.15$ and 1.09 with a sensitivity of $91.7 \%, 91.7 \%, 91.7 \%$ and specificity of $95.0 \%, 85.0 \%$ and $95.0 \%$, respectively. These findings can be explained by the high hypervascularization with leaky blood vessels inside WT (104), whereas PA is the less vascularized neoplasia because of the high content of mucus and few blood vessels. MTs show mostly peripheral phenomena of immature neovascularization, therefore quantitative DECT parameters proved to be intermediated between WTs and Pas. DECT is also used in radiotherapy treatment planning and in the evaluation after surgical or radiotherapy treatment (105) (Figure 4).

\section{Conclusion}

DECT provides helpful diagnostic tools for imaging gland tumours, without an additional or even lower radiation dose, compared to conventional CT. Low monochromatic images and iodine maps improve neoplastic lesion detection and conspicuity. Quantitative parameters, such as IC and HU slope, are extremely promising for lesion characterization, 
differential diagnosis and evaluation of response to treatment. Furthermore the sensitivity of DECT for iodine permits the reduction of amount and concentration of intravenous contrast media, particularly useful in patients at risk of contrast-induced nephropathy; finally the use of the high-energy (high $\mathrm{keV}$ ) data set can help in streak and bony artefact reduction.

\section{Acknowledgments}

Funding: None.

\section{Footnote}

Provenance and Peer Review: This article was commissioned by the Guest Editor (Antonio Barile) for the series "Multimodality Advanced Imaging and Intervention in Gland Diseases" published in Gland Surgery. The article has undergone external peer review.

Reporting Checklist: The authors have completed the NARRATIVE REVIEW reporting checklist. Available at http://dx.doi.org/10.21037/gs-20-543

Peer Review File: Available at http://dx.doi.org/10.21037/gs20-543

Conflicts of Interest: All authors have completed the ICMJE uniform disclosure form (available at http://dx.doi. org/10.21037/gs-20-543). The series "Multimodality Advanced Imaging and Intervention in Gland Diseases" was commissioned by the editorial office without any funding or sponsorship. The authors have no other conflicts of interest to declare.

Ethical Statement: The authors are accountable for all aspects of the work in ensuring that questions related to the accuracy or integrity of any part of the work are appropriately investigated and resolved.

Open Access Statement: This is an Open Access article distributed in accordance with the Creative Commons Attribution-NonCommercial-NoDerivs 4.0 International License (CC BY-NC-ND 4.0), which permits the noncommercial replication and distribution of the article with the strict proviso that no changes or edits are made and the original work is properly cited (including links to both the formal publication through the relevant DOI and the license). See: https://creativecommons.org/licenses/by-nc-nd/4.0/.

\section{References}

1. Agostini A, Borgheresi A, Mari A, et al. Dual-energy CT: theoretical principles and clinical applications. Radiol Med 2019;124:1281-95.

2. Johnson TR, Krauss B, Sedlmair M, et al. Material differentiation by dual energy CT: initial experience. Eur Radiol 2007;17:1510-7.

3. Carotti M, Salaffi F, Beci G, et al. The application of dual-energy computed tomography in the diagnosis of musculoskeletal disorders: a review of current concepts and applications. Radiol Med 2019;124:1175-83.

4. Biondi M, Vanzi E, De Otto G, et al. Water/cortical bone decomposition: A new approach in dual energy CT imaging for bone marrow oedema detection. A feasibility study. Phys Med 2016;32:1712-6.

5. Chiro GD, Brooks RA, Kessler RM, et al. Tissue signatures with dual-energy computed tomography. Radiology 1979;131:521-3.

6. Siegel MJ, Kaza RK, Bolus DN, et al. White Paper of the Society of Computed Body Tomography and Magnetic Resonance on Dual-Energy CT, Part 1: Technology and Terminology. J Comput Assist Tomogr 2016;40:841-5.

7. Foley WD, Shuman WP, Siegel MJ, et al. White Paper of the Society of Computed Body Tomography and Magnetic Resonance on Dual-Energy CT, Part 2: Radiation Dose and Iodine Sensitivity. J Comput Assist Tomogr 2016;40:846-50.

8. Graser A, Johnson TRC, Chandarana H, et al. Dual energy CT: preliminary observations and potential clinical applications in the abdomen. Eur Radiol 2009;19:13-23.

9. Brown CL, Hartman RP, Dzyubak OP, et al. Dual- energy CT iodine overlay technique for characterization of renal masses as cyst or solid: a phantom feasibility study. Eur Radiol 2009;19:1289-95.

10. Agrawal MD, Pinho DF, Kulkarni NM, et al. Oncologic applications of dual-energy CT in the abdomen. Radiographics. 2014;34:589-612.

11. Gentili F, Bronico I, Maestroni U, et al. Small renal masses $(\leq 4 \mathrm{~cm})$ : differentiation of oncocytoma from renal clear cell carcinoma using ratio of lesion to cortex attenuation and aorta-lesion attenuation difference (ALAD) on contrast-enhanced CT. Radiol Med 2020;125:1280-7.

12. Scialpi M, Volterrani L, Mazzei MA, et al. Small (< or $=2 \mathrm{~cm}$ ) atypical hepatic haemangiomas in the noncirrhotic patient: pattern-based classification scheme for enhancement at triple-phase helical CT. Radiol Med 2009;114:935-47. 
13. Hickethier T, Kroeger JR, Lennartz S, et al. Venous-phase chest $\mathrm{CT}$ with reduced contrast medium dose: Utilization of spectral low keV monoenergetic images improves image quality. Eur J Radiol 2020;122:108756.

14. Jamali S, Michoux N, Coche E, et al. Virtual unenhanced phase with spectral dual-energy CT: Is it an alternative to conventional true unenhanced phase for abdominal tissues? Diagn Interv Imaging 2019;100:503-11.

15. Cicero G, Ascenti G, Albrecht MH, et al. Extra-abdominal dual-energy CT applications: a comprehensive overview. Radiol Med 2020;125:384-97.

16. Bottari A, Silipigni S, Carerj ML, et al. Dual-source dual-energy CT in the evaluation of hepatic fractional extracellular space in cirrhosis. Radiol Med 2020;125:7-14.

17. Mazzei MA, Gentili F, Volterrani L. Dual-Energy CT Iodine Mapping and 40-keV Monoenergetic Applications in the Diagnosis of Acute Bowel Ischemia: A Necessary Clarification. AJR Am J Roentgenol 2019;212:W93-W94.

18. Mazzei MA, Guerrini S, Cioffi Squitieri N, et al. Diagnosis of acute mesenteric ischemia/infarction in the era of multislice CT. Recenti Prog Med 2012;103:435-7.

19. Mileto A, Marin D, Alfaro-Cordoba M, et al. Iodine quantification to distinguish clear cell from papillary renal cell carcinoma at dual-energy multidetector CT: a multireader diagnostic performance study. Radiology 2014;273:813-20.

20. Agostini A, Mari A, Lanza C, et al. Trends in radiation dose and image quality for pediatric patients with a multidetector CT and a third-generation dual-source dualenergy CT. Radiol Med 2019;124:745-52.

21. Yoshida R, Usui K, Katsunuma Y, et al. Reducing contrast dose using virtual monoenergetic imaging for aortic CTA. J Appl Clin Med Phys. 2020;10.1002/acm2.12951.

22. Si-Mohamed S, Dupuis N, Tatard-Leitman V, et al. Virtual versus true non-contrast dual-energy CT imaging for the diagnosis of aortic intramural hematoma. Eur Radiol 2019;29:6762-71.

23. Foti G, Beltramello A, Minerva G, et al. Identification of residual-recurrent cholesteatoma in operated ears: diagnostic accuracy of dual-energy CT and MRI. Radiol Med 2019;124:478-86.

24. Chawla A, Srinivasan S, Lim TC, et al. Dual-energy CT applications in salivary gland lesions. Br J Radiol 2017;90:20160859.

25. Sugawara H, Takayanagi T, Ishikawa T, et al. New Fast $\mathrm{kVp}$ Switching Dual-Energy CT: Reduced Severity of Beam Hardening Artifacts and Improved Image Quality in Reduced-Iodine Virtual Monochromatic Imaging. Acad
Radiol 2020;27:1586-93.

26. Dunnick NR, Korobkin M, Francis I. Adrenal radiology: distinguishing benign from malignant adrenal masses. AJR Am J Roentgenol 1996;167:861-7.

27. Young WF Jr. Clinical practice. The incidentally discovered adrenal mass. N Engl J Med 2007;356:601-10.

28. Foti G, Malleo G, Faccioli N, et al. Characterization of adrenal lesions using MDCT wash-out parameters: diagnostic accuracy of several combinations of intermediate and delayed phases. Radiol Med 2018;123:833-40.

29. Helck A, Hummel N, Meinel FG, et al. Can single-phase dual-energy CT reliably identify adrenal adenomas? Eur Radiol 2014;24:1636-42.

30. Botsikas D, Triponez F, Boudabbous S, et al. Incidental adrenal lesions detected on enhanced abdominal dualenergy CT: can the diagnostic workup be shortened by the implementation of virtual unenhanced images? Eur J Radiol 2014;83:1746-51.

31. Gupta RT, Ho LM, Marin D, et al. Dual-energy CT for characterization of adrenal nodules: initial experience. AJR Am J Roentgenol 2010;194:1479-83.

32. Siegel R, Naishadham D, Jemal A. Cancer statistics, 2012. CA Cancer J Clin 2012;62:10-29.

33. Witkowski ER, Smith JK, Tseng JF. Outcomes following resection of pancreatic cancer. J Surg Oncol 2013;107:97-103.

34. George E, Wortman JR, Fulwadhva UP, et al. Dual energy CT applications in pancreatic pathologies. Br J Radiol 2017;90:20170411.

35. El Kayal N, Lennartz S, Ekdawi S, et al. Value of spectral detector computed tomography for assessment of pancreatic lesions. Eur J Radiol 2019;118:215-22.

36. Bao J, Liu A, Zhao C, et al. Correlation Between DualEnergy Computed Tomography Single Scan and Computed Tomography Perfusion for Pancreatic Cancer Patients: Initial Experience. J Comput Assist Tomogr 2019;43:599-604.

37. Beer L, Toepker M, Ba-Ssalamah A, et al. Objective and subjective comparison of virtual monoenergetic vs. polychromatic images in patients with pancreatic ductal adenocarcinoma. Eur Radiol 2019;29:3617-25.

38. Bhosale P, Le O, Balachandran A, et al. Quantitative and Qualitative Comparison of Single-Source DualEnergy Computed Tomography and $120-k V p$ Computed Tomography for the Assessment of Pancreatic Ductal Adenocarcinoma. J Comput Assist Tomogr 2015;39:907-13.

39. Patel BN, Thomas JV, Lockhart ME, et al. Single-source dual-energy spectral multidetector $\mathrm{CT}$ of pancreatic 
adenocarcinoma: optimization of energy level viewing significantly increases lesion contrast. Clin Radiol 2013;68:148-54.

40. McNamara MM, Little MD, Alexander LF, et al. Multireader evaluation of lesion conspicuity in small pancreatic adenocarcinomas: complimentary value of iodine material density and low $\mathrm{keV}$ simulated monoenergetic images using multiphasic rapid $\mathrm{kVp}$-switching dual energy CT. Abdom Imaging 2015;40:1230-40.

41. Di Maso LD, Huang J, Bassetti MF, et al. Investigating a novel split-filter dual-energy CT technique for improving pancreas tumor visibility for radiation therapy. J Appl Clin Med Phys 2018;19:676-83.

42. Chu AJ, Lee JM, Lee YJ, et al. Dual-source, dual-energy multidetector CT for the evaluation of pancreatic tumours. Br J Radiol 2012;85:e891-8.

43. Ambrosio MR, Rocca BJ, Mastrogiulio MG, et al. Cystic gastrointestinal stromal tumors of the pancreas simulating cystoadenocarcinoma. Report of three cases and short review of the literature. Histol Histopathol 2014;29:1583-91.

44. Mazzei MA, Cioffi Squitieri N, Vindigni C, et al. Gastrointestinal stromal tumors (GIST): a proposal of a "CT-based predictive model of Miettinen index" in predicting the risk of malignancy. Abdom Radiol (NY) 2020;45:2989-96.

45. Morgan DE. Dual-energy CT of the abdomen. Abdom Imaging 2014;39:108-34.

46. Forstner R, Meissnitzer M, Cunha TM. Update on imaging of ovarian cancer. Curr Radiol Rep 2016;4:31.

47. Mazzei MA, Khader L, Cirigliano A, et al. Accuracy of MDCT in the preoperative definition of Peritoneal Cancer Index (PCI) in patients with advanced ovarian cancer who underwent peritonectomy and hyperthermic intraperitoneal chemotherapy (HIPEC). Abdom Imaging 2013;38:1422-30.

48. Roviello F, Pinto E, Corso G, et al. Safety and potential benefit of hyperthermic intraperitoneal chemotherapy (HIPEC) in peritoneal carcinomatosis from primary or recurrent ovarian cancer. J Surg Oncol 2010;102:663-70.

49. Pang T, Liu ZD, Deng K, et al. Preliminary application of multiple parameters spectral CT in the diagnosis of ovarian cancer. Medicine (Baltimore) 2017;96:e7786.

50. Thomassin-Naggara I, Bazot M, Daraï E, et al. Epithelial ovarian tumors: value of dynamic contrast-enhanced MR imaging and correlation with tumor angiogenesis. Radiology 2008;248:148-59.
51. Li GJ, Gao J, Wang GL, et al. Correlation between vascular endothelial growth factor and quantitative dualenergy spectral CT in non-small-cell lung cancer. Clin Radiol 2016;71:363-8.

52. Benveniste AP, de Castro Faria S, Broering G, et al. Potential Application of Dual-Energy CT in Gynecologic Cancer: Initial Experience. AJR Am J Roentgenol. 2017;208:695-705. Review. Erratum in: AJR Am J Roentgenol 2017;208:1176.

53. Yan WQ, Xin YK, Jing Y, et al. Iodine Quantification Using Dual-Energy Computed Tomography for Differentiating Thymic Tumors. J Comput Assist Tomogr 2018;42:873-80.

54. Chang S, Hur J, Im DJ, et al. Volume-based quantification using dual-energy computed tomography in the differentiation of thymic epithelial tumours: an initial experience. Eur Radiol 2017;27:1992-2001.

55. Gentili F, Pelini V, Lucii G, et al. Update in diagnostic imaging of the thymus and anterior mediastinal masses. Gland Surg 2019;8:S188-S207.

56. Khandelwal A, Sholl LM, Araki T, et al. Patterns of metastasis and recurrence in thymic epithelial tumours: longitudinal imaging review in correlation with histological subtypes. Clin Radiol 2016;71:1010-7.

57. Mazzei MA, Volterrani L. Errors in multidetector row computed tomography. Radiol Med 2015;120:785-94.

58. Mazzei MA, Contorni F, Gentili F, et al. Incidental and Underreported Pleural Plaques at Chest CT: Do Not Miss Them-Asbestos Exposure Still Exists. Biomed Res Int 2017;2017:6797826.

59. Mazzei MA, Sartorelli P, Bagnacci G, et al. Occupational Lung Diseases: Underreported Diagnosis in Radiological Practice. Semin Ultrasound CT MR 2019;40:36-50.

60. Paolucci V, Romeo R, Sisinni AG, et al. Silicosis in Workers Exposed to Artificial Quartz Conglomerates: Does It Differ From Chronic Simple Silicosis? Arch Bronconeumol 2015;51:e57-60.

61. Iannarelli A, Sacconi B, Tomei F, et al. Analysis of CT features and quantitative texture analysis in patients with thymic tumors: correlation with grading and staging. Radiol Med 2018;123:345-50.

62. Mazzei MA, Nardone V, Di Giacomo L, et al. The role of delta radiomics in gastric cancer. Quant Imaging Med Surg 2018;8:719-21.

63. Volterrani L, Mazzei MA, Fedi M, et al. Computed tomography perfusion using first pass methods for lung nodule characterization: limits and implications in radiologic practice. Invest Radiol 2009;44:124; author 
reply 124.

64. Mazzei MA, Preda L, Cianfoni A, et al. CT perfusion: technical developments and current and future applications. Biomed Res Int 2015;2015:397521.

65. Mazzei MA, Cioffi Squitieri N, Guerrini S et al. Quantitative CT perfusion measurements in characterization of solitary pulmonary nodules: new insights and limitations. Recenti Prog Med 2013;104:430-7.

66. Mazzei FG, Volterrani L, Guerrini S, et al. Reduced time CT perfusion acquisitions are sufficient to measure the permeability surface area product with a deconvolution method. Biomed Res Int 2014;2014:573268.

67. Mazzei FG, Mazzei MA, Cioffi Squitieri N, et al. CT perfusion in the characterisation of renal lesions: an added value to multiphasic CT. Biomed Res Int 2014;2014:135013.

68. Mazzei MA, Squitieri NC, Sani E, et al. Differences in perfusion CT parameter values with commercial software upgrades: a preliminary report about algorithm consistency and stability. Acta Radiol 2013;54:805-11.

69. Mazzei FG, Gentili F, Guerrini S, et al. MR Lymphangiography: A Practical Guide to Perform It and a Brief Review of the Literature from a Technical Point of View. Biomed Res Int 2017;2017:2598358.

70. Gennaro P, Borghini A, Chisci G, et al. Could MRI visualize the invisible? An Italian single center study comparing magnetic resonance lymphography (MRL), super microsurgery and histology in the identification of lymphatic vessels. Eur Rev Med Pharmacol Sci 2017;21:687-94.

71. Mazzei MA, Gentili F, Mazzei FG, et al. High-resolution MR lymphangiography for planning lymphaticovenous anastomosis treatment: a single-centre experience. Radiol Med 2017;122:918-27.

72. Gentili F, Guerrini S, Mazzei FG, et al. MRL as oneshot examination for patients suffering from lymphedema. Radiol Med 2020;125:798-9.

73. Guerrini S, Gentili F, Mazzei FG, et al. MR lymphangiography: with or without contrast? Biomed Res Int 2017;2017:2598358.

74. Hodgson NC, Button J, Solorzano CC. Thyroid cancer: Is the incidence still increasing? Ann Surg Oncol 2004;11:1093-7.

75. Gitto S, Grassi G, De Angelis C, et al. A computer-aided diagnosis system for the assessment and characterization of low-to-high suspicion thyroid nodules on ultrasound. Radiol Med 2019;124:118-25.

76. Ahn SS, Kim EK, Kang DR, et al. Biopsy of thyroid nodules: comparison of three sets of guidelines. AJR Am J Roentgenol 2010;194:31-7.

77. Aydin S, Fatihoglu, E, Kacar M. Intrathyroidal ectopic thymus tissue: a diagnostic challenge. Radiol Med 2019;124:505-9.

78. Ahn HS, Lee JB, Seo M, et al. Distinguishing benign from malignant thyroid nodules using thyroid ultrasonography: utility of adding superb microvascular imaging and elastography. Radiol Med 2018;123:260-70.

79. Kim DW, Lee YJ, Ahn HS, et al. Comparison of ultrasonography and computed tomography for diagnosing diffuse thyroid disease: a multicenter study. Radiol Med 2018;123:515-23.

80. Li HW, Wu XW, Liu B, et al. Clinical values of gemstone spectral CT in diagnosing thyroid disease. J Xray Sci Technol 2015;23:45-56.

81. Gao SY, Zhang XY, Wei W, et al. Identification of benign and malignant thyroid nodules by in vivo iodine concentration measurement using single-source dual energy CT: A retrospective diagnostic accuracy study. Medicine (Baltimore) 2016;95:e4816.

82. Lee DH, Lee YH, Seo HS et al. Dual-energy CT iodine quantification for characterizing focal thyroid lesions. Head Neck 2019;41:1024-31.

83. Liu X, Ouyang D, Li H, et al. Papillary thyroid cancer: dual-energy spectral CT quantitative parameters for preoperative diagnosis of metastasis to the cervical lymph nodes. Radiology 2015;275:167-76.

84. Miles KA. Tumour angiogenesis and its relation to contrast enhancement on computed tomography: a review. Eur J Radiol 1999;30:198-205.

85. Seyednejad N, Healy C, Tiwari P, et al. Dualenergy computed tomography: a promising novel preoperative localization study for treatment of primary hyperparathyroidism. Am J Surg 2016;211:839-45.

86. Johnson TRC. Dual energy CT: general principles. AJR Am J Roentgenol 2012;199:S3-8.

87. Karçaaltıncaba M, Aktas A. Dual-energy CT revisited with multidetector CT: review of principles and clinical applications. Diagn Interv Radiol 2011;17:181-94.

88. Roskies M, Liu X, Hier MP, et al. 3-phase dual-energy CT scan as a feasible salvage imaging modality for the identification of non-localizing parathyroid adenomas: a prospective study. J Otolaryngol Head Neck Surg 2015;44:44.

89. Moon JI, Choi BH, Baek HJ, et al. Comprehensive analyses with radiological and biological markers of breast cancer on contrast-enhanced chest CT: a single center 
experience using dual-layer spectral detector CT. Eur Radiol 2020;30:2782-90.

90. Inoue T, Nakaura T, Iyama A, et al. Usefulness of Virtual Monochromatic Dual-Layer Computed Tomographic Imaging for Breast Carcinoma. J Comput Assist Tomogr 2020;44:78-82.

91. Okada K, Matsuda M, Tsuda T, et al. Dual-energy computed tomography for evaluation of breast cancer: value of virtual monoenergetic images reconstructed with a noise-reduced monoenergetic reconstruction algorithm. Jpn J Radiol 2020;38:154-64.

92. Metin Y, Metin NO, Özdemir O, et al. The role of low $\mathrm{keV}$ virtual monochromatic imaging in increasing the conspicuity of primary breast cancer in dual-energy spectral thoracic CT examination for staging purposes. Acta Radiol 2020;61:168-74.

93. Volterrani L, Gentili F, Fausto A, et al. Dual-Energy CT for Locoregional Staging of Breast Cancer: Preliminary Results. AJR Am J Roentgenol 2020;214:707-14.

94. Yoshida T, Urikura A, Shirata K, et al. Short tau inversion recovery in breast diffusion-weighted imaging: signalto-noise ratio and apparent diffusion coefficients using a breast phantom in comparison with spectral attenuated inversion recovery. Radiol Med 2018;123:296-304.

95. Ecanow JS, Abe H, Newstead GM, et al. Axillary staging of breast cancer: what the radiologist should know. RadioGraphics 2013;33:1589-612.

96. Setacci F, Sirignano P, de Donato G, et al. Two-yearresults of Endurant stent-graft in challenging aortic neck morphologies versus standard anatomies. J Cardiovasc Surg (Torino) 2014;55:85-92.

97. Zhang X, Zheng C, Yang Z, et al. Axillary Sentinel Lymph Nodes in Breast Cancer: Quantitative Evaluation at Dual-

Cite this article as: Gentili F, Guerrini S, Mazzei FG, Monteleone I, Di Meglio N, Sansotta L, Perrella A, Puglisi S, De Filippo M, Gennaro P, Volterrani L, Castagna MG, Dotta F, Mazzei MA. Dual energy CT in gland tumors: a comprehensive narrative review and differential diagnosis. Gland Surg 2020;9(6):2269-2282. doi: 10.21037/gs-20-543
Energy CT. Radiology 2018;289:337-46.

98. Bruno F, Arrigoni F, Mariani S, et al. Advanced magnetic resonance imaging (MRI) of soft tissue tumors: techniques and applications. Radiol Med 2019;124:243-52.

99. Hisatomi M, Asaumi J, Yanagi Y, et al. Diagnostic value of dynamic contrast-enhanced MRI in the salivary gland tumors. Oral Oncol 2007;43:940-7.

100. Vogl TJ, Albrecht MH, Nour-Eldin NA, et al. Assessment of salivary gland tumors using MRI and CT: impact of experience on diagnostic accuracy. Radiol Med 2018;123:105-16.

101. Kim DW. Computed tomography features of the major salivary glands after radioactive iodine ablation in patients with papillary thyroid carcinoma. Radiol Med 2018;123:20-7.

102. Nardone V, Tini P, Nioche C, et al. Texture analysis as a predictor of radiation-induced xerostomia in head and neck patients undergoing IMRT. Radiol Med 2018;123:415-23.

103. Li L, Zhao Y, Luo D, et al. Diagnostic value of singlesource dual-energy spectral computed tomography in differentiating parotid gland tumors: initial results. Quant Imaging Med Surg 2018;8:588-96.

104. Woo SH, Choi DS, Kim JP, et al. Two-phase computed tomography study of warthin tumor of parotid gland: differentiation from other parotid gland tumors and its pathologic explanation. J Comput Assist Tomogr 2013;37:518-24.

105. Torroni A, Gennaro P, Aboh IV, Longo G, Valentini V, Iannetti G. Microvascular reconstruction of the mandible in irradiated patients. J Craniofac Surg 2007;18:1359-69. 